\title{
ON SOME THEOREMS IN THE LIE THEORY.
}

BY PROFESSOR L. D. AMES.

(Read before the Southwestern Section of the American Mathematical Society, November 27, 1909.)

THE well-known treatment of the infinitesimal transformation in the Lie theory makes use of power series, assuming that the functions involved are analytic. The treatment here given demands at most the existence of second partial derivatives. Since no use is made of the group theory, the proofs here given may be used either in connection with the group theory or apart from it.

Three theorems are stated in this paper, of which Theorem I. was proved in a previous paper.* Of the three, any one is an immediate result of the other two taken together. They may be compactly stated as follows:

Given any differential equation of the form

$$
\Omega\left(x, y, y^{\prime}\right)=0
$$

which, in a given region $R$, can be written in the form linear in $y^{\prime}$

$$
\Omega_{1} \equiv X(x, y) y^{\prime}-Y(x, y)=0,
$$

and of which $\omega(x, y)=c$ is an integral; let $\xi(x, y)$ and $\eta(x, y)$ be such functions that

$$
X \eta-Y \xi \neq 0 .
$$

Consider the statements

A. The equation

$$
\frac{X}{X \eta-Y \xi} y^{\prime}-\frac{Y}{X \eta-Y \xi}=0
$$

is exact. That is, $1 /(X \eta-Y \xi)$ is an integrating factor of (2). B.

$$
\xi \frac{\partial \Omega}{\partial x}+\eta \frac{\partial \Omega}{\partial y}+\eta^{\prime} \frac{\partial \Omega}{\partial y^{\prime}}=0, \dagger
$$

* Bulletin, vol. 15, no. 8 (May, 1909).

$\dagger$ In the Lie theory this is the condition for invariance under the extended transformation

$$
U^{\prime} f=\xi \frac{\partial f}{\partial x}+\eta \frac{\partial f}{\partial y}+\eta^{\prime} \frac{\partial f}{\partial y^{\prime}} .
$$


where

$$
\eta^{\prime}=\frac{d \eta}{d x}-y^{\prime} \frac{d \xi}{d x}
$$

C.

$$
\xi \frac{\partial \omega}{\partial x}+\eta \frac{\partial \omega}{\partial y}=F(\omega) *
$$

THEOREM I. B is a necessary and sufficient condition for $\mathbf{A}$.

THEOREM II. C is anecessary and sufficient condition for $\mathrm{B}$.

THEOREM III. C is a necessary and sufficient condition for A.

The proofs are in outline as follows :

Theorem I. The familiar condition that (3) be exact, after obvious simplifications, is (4).

Theorem II. Differentiate (5) totally along $\omega=c$. The result, after obvious substitutions, is (4).

Theorem III. Assume that $M$ is the unknown integrating factor of (2). Writing the exact equation in two forms, we obtain an equation which we can solve for $M$. This proof does not assume a previous knowledge of the form of the integrating factor.

More detailed proofs of Theorems II. and III. follow.

Proof of Theorem II. First to prove $C$ sufficient. Since $\omega=c$ is an integral of (2) we may write (2) in the form

$$
\Omega_{1} \equiv M \frac{\partial \omega}{\partial x}+M y^{\prime} \frac{\partial \omega}{\partial y}=0
$$

As in the previous paper, the partial derivatives of $\Omega$ with respect to $x, y$ and $y^{\prime}$ are proportional to those of $\Omega_{1}$. Form these and substitute in the equation obtained by differentiating (5) totally along the curve $\omega=c$. The result is (4).

To prove $C$ necessary we assume (4), and retrace the steps of

* In the lie theory this is the condition that the family of integral curves $\omega(x, y)=c$ is invariant under the transformation

$$
U f=\xi \frac{\partial f}{\partial x}+\eta \frac{\partial f}{\partial y}
$$

Throughout this paper $F(\omega)$ is to be read "some function of $\omega$ " when (5) is a necessary condition, and "any function of $\omega$ " when (5) is a sufficient condition. 
the above proof, obtaining

$$
\frac{d}{d x}\left[\xi \frac{\partial \omega}{\partial x}+\eta \frac{\partial \omega}{\partial y}\right]=0 .
$$

Noting that $y$ and $y^{\prime}$ are functions of $x$ and $c$, the integration of (7) gives

$$
\xi \frac{\partial \omega}{\partial x}+\eta \frac{\partial \omega}{\partial y}=F(c)=F(\omega)
$$

Proof of Theorem III. First to prove $C$ sufficient. Equation (2) has an integrating factor which we will call $M$. Then the equation

$$
M X y^{\prime}-M Y=0
$$

is exact. It is therefore of the form

$$
\frac{\partial \omega_{1}}{\partial y} y^{\prime}+\frac{\partial \omega_{1}}{\partial x}=0
$$

where $\omega$ is some function of $\omega_{1}, \omega=\phi\left(\omega_{1}\right)$. Hence

$$
\begin{aligned}
& \frac{\partial \omega}{\partial x}=\phi^{\prime}\left(\omega_{1}\right) \frac{\partial \omega_{1}}{\partial x}=-\phi^{\prime}\left(\omega_{1}\right) M Y, \\
& \frac{\partial \omega}{\partial y}=\phi^{\prime}\left(\omega_{1}\right) \frac{\partial \omega_{1}}{\partial y}=\phi^{\prime}\left(\omega_{1}\right) M X .
\end{aligned}
$$

Putting these values in (5) and solving for $M$, we obtain

$$
M=\psi\left(\omega_{1}\right) /(X \eta-Y \xi),
$$

where $\psi$ is some function of $\omega_{1}$. Hence $1 /(X \eta-Y \xi)$ is also an integrating factor.

To prove that (5) is necessary, let $1 /(X \eta-Y \xi)$ be a particular integrating factor, and $\omega_{1}(x, y)=c$ the corresponding integral. Let $\omega(x, y)=c$ be any integral. Then the corresponding integrating factor is $\chi\left(\omega_{1}\right) /(X \eta-Y \xi)$, where $\chi$ is some function of $\omega_{1}$. Hence the equations

$$
\frac{X \chi}{X \eta-Y \xi} y^{\prime}-\frac{Y \chi}{X \eta-Y \xi}=0
$$


and

are identical. Hence

$$
\frac{\partial \omega}{\partial y} y^{\prime}+\frac{\partial \omega}{\partial x}=0
$$

$$
\xi \frac{\partial \omega}{\partial x}+\eta \frac{\partial \omega}{\partial y}=\chi\left(\omega_{1}\right)=F(\omega) .
$$

University of Missouri, November, 1909.

\section{ON THE DISCONTINUOUS $\zeta$-GROUPS DEFINED BY RATIONAL NORMAL CURVES IN A SPACE OF $n$ DIMENSIONS.}

BY PROFIGSSOR J. W. YOUNG.

(Read before the Chicago Section of the American Mathematical Society, January 1, 1910.)

THE present note completes in an important particular a paper* which I presented to the Society some two years ago. I there considered the discontinuous groups $\Gamma_{n}$ of linear fractional transformations

$$
\zeta^{\prime}=\begin{aligned}
& \alpha \zeta+\beta \\
& \gamma \zeta+\delta
\end{aligned}
$$

on the complex variable $\zeta$, defined as follows by a rational normal curve $C_{n}$ in a space $S_{n}$ of $n$ dimensions : The given $C_{n}$ is transformed into itself by a group of $\infty^{3}$ collineations

$$
z_{i}^{\prime}=\sum_{k=0}^{n} a_{i k_{k} z_{k}} \quad(i=0,1, \ldots, n)
$$

in $S_{n}$. Each of these collineations subjects the parameter $\zeta$ of the points of $C_{n}$ to a substitution (1), so that the continuous three-parameter groups of transformations (1) and (2) are simply isomorphic. If now the transformations (2) be restricted to those whose coefficients $a_{i k}$ are rational integers with determinant $\left|a_{i k_{f}}\right|=1$, the resulting subgroup of the three-parameter group of transformations (2) will be properly discontinuous.

* " A fundamental invariant of the discontinuous $\zeta$-groups defined by the normal curves of order $n$ in a space of $n$ dimensions," BuLceTrN, vol. 14 (1908), pp. 363-367. 